\title{
UTILIDAD DE LOS MARCADORES TUMORALES EN EL PRONÓSTICO DEL CÁNCER TRANSICIONAL DE VEJIGA
}

\author{
M.F. LORENZO GÓMEZ, G. SCHROEDER \\ Department of Urology. University of Miami. Miami. Florida. USA.
}

Actas Urol Esp. 27 (7): 501-512, 2003

\section{RESUMEN}

UTILIDAD DE LOS MARCADORES TUMORALES EN EL PRONÓSTICO DEL CÁNCER TRANSICIONAL DE VEJIGA

OBJETIVOS DEL TRABAJO: Revisión del estado actual de los marcadores tumorales disponibles para el pronóstico y exposición de los problemas con el grado y el estadiaje local del cáncer transicional de vejiga.

PROCEDIMIENTOS BÁSICOS: Revisión de la literatura publicada en marcadores de cáncer transicional de vejiga mediante una búsqueda en Medline y análisis crítico de la misma.

RESULTADOS: Hay diferencias estadísticamente significativas interobservador en la determinación del grado. Hay un nuevo sistema de combinación de grados. Existen problemas en la determinación del estadio local y baja correspondencia entre el estadio clínico y el patológico post-cistectomía radical. Los principales marcadores tumorales pronósticos considerados en este cáncer son citometría de flujo, estudio cariocitométrico, oncogenes (p53, bcl-2, Her2/Neu o c-erbB2), anomalías cromosómicas (cromosoma 9, cromosomas 7 y 17), marcadores de proliferación (Ki-

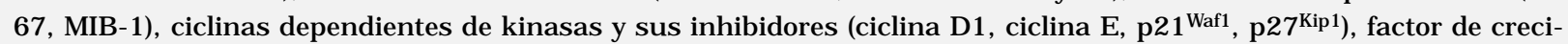
miento endotelial vascular, otros factores de crecimiento (fibroblástico, epidérmico, hepatocitario, derivado de plaquetas), metaloproteinasas, moléculas de adhesióny otros.

CONCLUSIONES: Actualmente, no hay un marcador tumoral pronóstico para el cáncer de vejiga superior al grado y estadio convencionales, a pesar de sus deficiencias. Se necesita una estandarización de los métodos de determinación de algunos marcadores que aporte resultados concluyentes y útiles en el terreno clínico. Algunas contradicciones en los resultados obtenidos por diferentes autores obligan a cuestionar el significado pronóstico de ciertos marcadores tumorales en el cáncer transicional de vejiga.

PALABLAS CLAVE: Vejiga. Tumor. Marcadores pronóstico.

\section{ABSTRACT \\ THE ROLE OF TUMOR MARKERS IN PROGNOSING TRANSITIONAL BLADDER CANCER}

PURPOSE: Review the literature on tumor markers used for prognosis of transitional bladder cancer. The existing problems regarding grading and local staging of these tumors are also discussed.

METHODS: The published literature on transitional bladder cancer markers was identified using a Medline search and critically analysed.

RESULTS: There are significant interobserver differences in grading. There is a new grading system. There are also problems in local staging and low correlation between clinic stage and pathologic stage. Major tumor markers studied for prognosis of transitional bladder cancer are: flow cytometry, kariocytometric study, oncogenes (p53, bcl-2, Her2/Neu or c-erbB2), chromosomic alterations (chromosomes 9, 7 and 17), proliferation markers (Ki-67, MIB-1), cyclin-dependent kinases and its inhibitors (cyclin D1, cyclin E, p2 $1^{\text {Wafl }}$, p2 $7^{\mathrm{Kipl}}$ ), vascular endothelial growth factor, other growth factors (fibroblastic, epidermal, hepatocyte, platelet-derived), metalloproteinases, cell adhesion molecules, and others.

CONCLUSIONS: At present, there are no prognostic markers for bladder cancer that are superior to conventional grading and staging, despite its imperfections. Standarization of assay methods in bladder tumor markers is needed to permit more conclusive and reproducible results and become a clinic tool. Controversy resulting from several studies make the meaning of some putative prognostic markers in transitional bladder cancer questionable.

KEY WORDS: Bladder. Tumor. Marker prognosis. 
$M^{2}$ ás del 90\% de los tumores de vejiga son carcinomas de células transicionales, y nos referiremos a ellos como cáncer de vejiga en este artículo. El grado tumoral, el estadio y la presencia de carcinoma in situ son los parámetros fundamentales utilizados para pronosticar el cáncer de vejiga. Sin embargo, por una parte estos parámetros dependen del espécimen y de la habilidad del patólogo, habiendo actualmente controversia en la interpretación tanto del grado como del estadio. Por otra parte, el cáncer vesical puede tener diferente comportamiento en diferentes pacientes aún presentándose en el mismo grado y estadio. Por lo tanto, un marcador molecular que pudiera predecir con precisión la posibilidad de recurrencia, el riesgo de progresión y la probable supervivencia en el paciente con cáncer vesical serían de gran utilidad. Tal marcador permitiría individualizar el tratamiento en base a características moleculares de cada tumor. Diversas moléculas y características de las células tumorales, como el p53, Ki-67, Rb, pérdida de heterozigosidad en el cromosoma 9, p-27, ciclina D1, E-cadherina, uroplakin y otras, están en estudio para poder ser útiles herramientas pronósticas. Además, algunos marcadores se enfocan a la predicción de respuesta al tratamiento con BCG, radioterapia, quimioterapia (p53, Ki-67, p27).

En este artículo presentamos una actualización y análisis de los marcadores tumorales disponibles que intentan ser una herramienta práctica que mejore nuestro conocimiento en el pronóstico del cáncer vesical, así como se reconoce la problemática en la determinación del grado y el estadio local de esta enfermedad.

\section{MATERIAL Y MÉTODOS}

Se ha procedido a una revisión de la literatura publicada en los marcadores tumorales relacionados con el pronóstico del cáncer de vejiga y a los estudios que aportan luz sobre la controversia en la determinación de grado y estadio local. Se realizó una búsqueda en Medline y se realizó su análisis crítico. El tipo de estudio realizado para cada marcador, tamaño muestral, reproductibilidad del procedimiento, tipo de procesamiento para la determinación de cada marcador, la disponibilidad real para llevarlo a la práctica en el medio clínico se han tenido en con- sideración, asi como también se han confrontado los resultados contradictorios encontrados por los autores.

\section{RESULTADOS Y DISCUSIÓN}

A. Controversia en los parámetros clínico-patológicos en el pronóstico del cáncer vesical.

B. Descripción y análisis de los principales marcadores tumorales pronósticos en el cáncer de vejiga.

C. Otros marcadores estudiados para el pronóstico del cáncer de vejiga.

D. Capacidad de los marcadores tumorales para predecir la respuesta al tratamiento.

\section{A. CONTROVERSIA EN LOS PARÁMETROS CLÍ-} NICO-PATOLÓGICOS EN EL PRONÓSTICO DEL CÁNCER VESICAL.

Aunque en general se acepta que la edad y el sexo no influyen en la historia natural ni en la distribución del estadio clínico del cáncer vesi$\mathrm{cal}^{1}$, en el proyecto Eurocare se encontró que el riesgo de muerte por este cáncer era significativamente superior en las mujeres. Este riesgo se atribuyó a un estadio más avanzado en el momento del diagnóstico ${ }^{2}$. Es posible que el atribuir los síntomas y signos del tumor a patologías femeninas benignas contribuya a este retardo en el diagnóstico. Algunos estudios de hibridación fluorescente in situ (FISH, ver más adelante) han encontrado una incidencia de aneusomía de cromosomas 7 y 17 significativamente más alta en los pacientes más jóvenes comparados con los de más edad ${ }^{3}$ pero este hallazgo no se ha traducido con diferencias en el pronóstico.

El grado histológico tumoral se ha considerado el factor pronóstico más importante en la progresión del cáncer. Algunos autores lo han encontrado el factor más correlacionado con el estadio y el tamaño del tumor ${ }^{1}$, mientras que en algunos estudios de pacientes cistectomizados se encontró que el grado no se relacionaba ni con el estadio patológico, ni con la presencia de metástasis linfáticas ni con la supervivencia ${ }^{4,5}$. Asímismo, hay estudios que no han encontrado asociación significativa entre el grado y el incremento del grado ni el estadio en la recurrencia en pacientes con tumores en estadios Ta/T1 en la presentación $^{6}$. El sistema clásico de gradación se 
basa en el grado de diferenciación de las células tumorales, y agrupa a los carcinomas en tres grados: bien, moderada y pobremente diferenciados, correspondiendo a grados 1,2 y 3 respectivamente $^{7}$. Sin embargo, el estudio al microscopio convencional está limitado por la apariencia citológica normal de los tumores bien diferenciados. Además, las células de estos cánceres son más cohesivas y no se desprenden a la orina, lo cual explica la mayor sensibilidad de la citología para los tumores de alto grado y el carcinoma in situ (CIS). Aun así, incluso en los tumores de alto grado se ha encontrado que la citología urinaria puede ser falso negativo hasta en un $20 \%$ de casos $^{8}$. Un resultado falso positivo en la citología puede ocurrir hasta en un $1-12 \%$ de pacientes debido a la atipia urotelial por inflamación o por cambios debidos a radioterapia o quimioterapia ${ }^{9}$. Por otra parte, se han encontrado diferencias estadísticamente significativas interobservador en la determinación del grado del tumor hasta en un $39 \%$ de $\operatorname{casos}^{10}$. Algunos autores consideran el bajo grado una condición para indicar tratamiento que preserve la vejiga (mediante resección transuretral, radio y quimioterapia) en casos seleccionados de cáncer vesical músculo invasi$v^{11}$. Aunque la clasificación de la Organización Mundial de la Salud y la Sociedad Internacional de Patología Urológica (WHO/ISUP) de 1998 y la tradicional del sistema de tres grados del espécimen de biopsia, se han considerado buenos predictores del grado histológico final del tumor, algunos estudios encuentran que la biopsia preoperatoria tiende a infraestadiar el cáncer ${ }^{12}$. En medio de toda esta controversia, WHO/ISUP han propuesto un nuevo sistema de gradación que tiene en cuenta la heterogeneidad patológica del cáncer de vejiga, aplicando una combinación de un grado primario (el más frecuente) y uno secundario (el segundo más frecuente). Los primeros estudios concluyen que la precisión diagnóstica mejora aplicando este nuevo sistema ${ }^{13}$.

En cuanto a la presencia de carcinoma in situ (CIS), en general se considera que influencia la recurrencia, progresión y mortalidad específica debida al cáncer de vejiga, aún en el superficial ${ }^{14}$. Se ha llegado a considerar que aún en el estadio T1, el CIS representa un cáncer más agresivo que ha de hacer considerar un tratamiento definitivo precoz ${ }^{15}$.
El estadio ha sido considerado el parámetro más importante para predecir la evolución clínica de los pacientes con cáncer de vejiga ${ }^{16}$. La afectación de la lámina propia (estadio T1) se ha asociado con la proporción de recurrencia y progresión ${ }^{17}$. Controvertidamente, otros estudios encuentran que el estadio no influencia la evolución del tumor y la profundidad de la afectación de la lámina propia no se encontró un factor pronóstico adverso significativo, ni para la progresión de la enfermedad ni para la supervivencia $^{14,15}$. El estadiaje en si mismo puede ser dificultoso. Se ha advertido la posibilidad de confusión de la túnica muscularis mucosa de la lámina propia con el músculo detrusor ${ }^{18}$. Asímismo, el tejido adiposo de la muscularis propia puede ser erróneamente considerado tejido perivesical en los especímenes de la resección transuretral de los tumores vesicales, lo que conduce al sobreestadiaje $^{19}$. Pérez Huguet y colegas, en una serie de 384 cistectomías radicales, encontraron correspondencia entre el estadio clínico y los hallazgos patológicos en un $32,5 \%$, sobre-estadiaje en un $28 \%$ e infraestadiaje en $39,5 \%$ de $\operatorname{casos}^{20}$. También otros autores encontraron infraestadiados hasta un $33 \%$ de tumores de grado alto o intermedio ${ }^{17}$. Este infraestadiaje conduce a una indicación errónea de preservar la vejiga, en vez de una cistectomía radical, que conlleva progresión de la enfermedad y peor pronóstico, mientras que el sobre-estadiaje conduce a cirugía, radioterapia o tratamiento citostático innecesarios.

\section{B. DESCRIPCIÓN Y ANÁLISIS DE LOS PRINCI- PALES MARCADORES TUMORALES PRO- NÓSTICOS EN EL CÁNCER DE VEJIGA.}

La citometría de flujo mide el contenido de ADN de las células. Cuantifica la población de células aneuploides ${ }^{8}$. Aunque en principio se consideró que la ploidía de ADN podría incrementar el valor predictivo de la citología de orina convencional ${ }^{21}$ posteriormente se ha demostrado que la sensibilidad respecto a la citología es sólo marginalmente superior y no se justifica su utilización ${ }^{22}$. La sensibilidad de la citometría de flujo puede incrementarse mediante técnicas de hibridación fluorescente in situ (FISH), que permiten una mejor detección de aberraciones cuantitativas de $\mathrm{ADN}^{25}$. 
Sin embargo, no se ha establecido de forma concluyente su utilidad en la práctica clínica.

La morfometría nuclear puede ser estudiada mediante el Quanticyt $\AA$, que es un sistema cariométrico que analiza cuantitativamente dos características nucleares: un índice de desviación llamado 2cDI y la forma nuclear promedio, en las células obtenidas mediante lavado vesical. Un 2cDI de 2,00 o más alto se ha encontrado asociado significativamente con CIS, cáncer invasivo y progresión ${ }^{24}$. Sin embargo, aunque la morfometría nuclear podría parecer asociada al grado histológico y a la actividad proliferativa, se ha encontrado que no tiene valor en la predicción del comportamiento clínico del cáncer vesical transicional ${ }^{25}$. Controvertidamente, Fujikawa encontró que el volumen nuclear medio fue el único factor pronóstico en el cáncer transicional en el tracto urinario superior, más que el grado o el estadio ${ }^{26}$.

p53 es una fosfoproteína que se une al ADN, codificada por el gen $17 \mathrm{p} 13.1^{27}$. Ha sido considerada un factor independiente relacionado con la supervivencia, progresión y desarrollo de metástasis en el cáncer de vejiga. Varios autores han encontrado diferencias significativas en la supervivencia libre de enfermedad entre los tumores T1G3 con inmunorreactividad p53(+) y p53(-) ${ }^{28-}$ 30. También la inmunotinción p53 se encontró asociada significativamente con la recurrencia y la progresión en tumores en estadio $\mathrm{T}^{31}$. Sin embargo, aunque a un nivel umbral o de corte de sobre-expresión de p53 del $20 \%$ se encontró la más alta correspondencia entre el pronóstico y la mutación p53, esta asociación no se ha encontrado superior al estadio TNM como factor pronóstico en otros estudios ${ }^{32}$. En muchos estudios no se ha encontrado útil la inmunorreactividad p53 en la caracterización de los cánceres transicionales de vejiga ${ }^{15,33-35}$. Asi mismo, la supervivencia libre de recurrencia no se ha demostrado asociada con el estatus p53 previo ${ }^{36,37}$. También se ha encontrado contradictoria la relación entre la mutación p53 y la expresión de la proteína p53. Las discordancias se han explicado en parte por la utilización de métodos inmunohistoquímicos diferentes ${ }^{38,39}$.

$b c l-2$ es un protooncogen que codifica una proteína de la membrana mitocondrial que blo- quea la apoptosis, por lo tanto es un gen antiapoptosis ${ }^{27}$. La sobre-expresión de bcl-2 citoplasmática se ha encontrado en la mayoría de cánceres transicionales de vejiga en pacientes más jóvenes, sin que se encontrara una asociación significativa con mayor tendencia a la recurrencia o a la progresión ${ }^{40}$. Mientras que algunos autores encuentran que un bcl-2 positivo se asocia significativamente a un descenso de supervivencia libre de tumor ${ }^{28}$, otros encuentran que la expresión positiva de bcl-2 y MST1/p16 detectados por inmunohistoquímica está incrementada en los cánceres vesicales primarios de grado y estadio más bajos y asociados con mejor pronóstico $^{41}$

Her2/Neu (también llamado c-erbB2 y p185) es un protooncogen que está en las células normales, localizado en 17q12-21.32 $2^{27}$. Mientras que algunos autores han encontrado asociación significativa entre la amplificación de c-erb-2 y un peor pronóstico en pacientes con tumores de alto grado y/o invasivos ${ }^{42} \mathrm{y}$ predictor de recurrencia a los 5 años de media de seguimiento ${ }^{43}$, otros autores obtienen resultados que no confirman esta asociación $^{44}$.

Anomalias en el cromosoma 9: Se encontró asociación significativa entre delecciones en las regiones 9ptr-22, 9q33 y 9q34 y la probabilidad de recurrencia ${ }^{45}$. Del mismo modo la pérdida de heterozigosidad (LOH) en 9q34 se asoció significativamente a cánceres de vejiga superficiales con más alto riesgo de recurrencia ${ }^{46}$. También la delección del locus TSC1 (situado en el cromosoma 9) se ha asociado significativamente con mayor riesgo de recurrencia ${ }^{47}$.

La aneusomía de los cromosomas 7 y 17, medida mediante FISH, se ha encontrado asociada significativamente con recurrencia en tumores $\mathrm{Ta} / \mathrm{T} 1$ en el primer diagnóstico ${ }^{6}$.

Marcadores de proliferación celular (Ki-67, MIB1) y combinaciones de los mismos: el Ki-67 es un anticuerpo monoclonal que reconoce a un antígeno nuclear humano expresado en las fases S, G1, G2 y M del ciclo celular; MIB-1 es un anticuerpo monoclonal equivalente del Ki-67 ${ }^{27}$. El índice de marcaje MIB1/Ki-67 se ha encontrado predictor de recurrencia tanto en cánceres vesicales de bajo grado, como en estadios Ta/T1 $1^{48,49}$. En combinación con otros marcadores tumorales de veji- 
ga (ver más adelante) Sgambato ha encontrado la existencia de un perfil consistente en alta expresión de Ki-67 (>10\% de núcleos positivos)/baja expresión de ciclina D1/baja expresión de p27 kip1 asociado significativamente a mayor riesgo de recurrencia en tumores vesicales primariamente en estadio $\mathrm{Ta} / \mathrm{T} 1^{50}$. También el perfil combinado alta expresión de Ki-67/baja de p27, medido por inmunohistoquímica, en tumores invasivos, se ha encontrado asociado significativamente a una peor supervivencia ${ }^{51}$.

$p 21^{\text {Waf } 1}$ y $p 27^{\text {Kip } 1}$ son dos inhibidores de las ciclinas dependientes de kinasas. Ambos son potentes reguladores negativos del ciclo celular. Su expresión reducida se encontró asociada significativamente con cánceres vesicales papilares superficiales con un curso más agresivo ${ }^{50}$. Otros autores encuentran que aunque hasta un $36 \%$ de tumores vesicales en estadios $\mathrm{Ta}$ y $\mathrm{T} 1$ sobreexpresan $\mathrm{p} 21$, este hecho no se asocia significativamente con los indicadores biológicos de la evolución clínica ${ }^{52}$. Así mismo, otros estudios no han encontrado asociación significativa entre la expresión de p2 $1^{\text {Wafl }}$ y la supervivencia libre de enfermedad $^{53}$. Para mayor confusión, en los tumores invasivos, la pérdida de expresión de p21 no se encontró asociada significativamente a una supervivencia libre de enfermedad acorta$\mathrm{da}^{54}$. Un estudio ha encontrado asociación significativa entre los niveles de p27 y de ciclina $E$ descendidos y tumores que progresaron a enfermedad invasiva ${ }^{55}$. La expresión de ciclina D1 medida por inmunohistoquímica, en tumores en estadios $\mathrm{Ta} / \mathrm{T} 1$, se ha encontrado un indicador de invasividad pero sin llegar a demostrarse como un factor pronóstico independiente ${ }^{56}$.

La doble tinción antígeno de la región del organizador nucleolar (AgNOR) y MIB-1 negativo, llamada también "resting NOR" o NOR en descanso (dado que MIB-1 se correlaciona con la actividad proliferativa), se ha encontrado asociada significativamente con un aumento de la existencia de metástasis a distancia y un peor pronóstico ${ }^{57,58}$.

Factor de crecimiento endotelial vascular (VEGF) y otros marcadores microvasculares: Mientras que algunos autores han encontrado VEGF, determinado por inmunotinción, asociado significativamente con la progresión del cáncer vesical $^{59}$ y a un nivel de corte de $400 \mathrm{pg} / \mathrm{ml}$ podría ser considerado útil para identificar cáncer vesical metastático ${ }^{60}$, otros autores no han encontrado asociación significativa ni con el riesgo de recurrencia, ni con la supervivencia del paciente en cánceres vesicales en estadios $\mathrm{Ta} / \mathrm{T}^{61}$. Mientras que la expresión de VEGFmRNA se ha encontrado 3 veces más alta en tumores vesicales superficiales, comparado con invasivos, no se ha encontrado diferencia significativa en la expresión de proteína VEGF. Por otra parte, en cánceres vesicales superficiales se ha encontrado asociación significativa entre una alta expresión de factor de iniciación eucariótico $4 \mathrm{E}$ (un factor implicado en la regulación translacional de VEGF) y un peor pronóstico ${ }^{62}$.

La densidad microvascular identificada por inmunotinción de CD31 de las células endoteliales, se asoció a metástasis en los nódulos linfáti$\cos$ y a la muerte del paciente tras una recurrencia pelviana ${ }^{63,64}$. Controvertidamente, la evaluación estereológica de la angiogénesis por marcaje CD31 del endotelio, no se ha encontrado útil para predecir la recurrencia en tumores vesicales superficiales $^{65}$.

Los niveles urinarios del factor del crecimiento fibroblástico básico (bFGF) se han encontrado altos en pacientes con tumores vesicales, pero este hallazgo no es específico de los tumores vesicales ${ }^{66}$. Van Rhijn propuso que en pacientes con cáncer vesical superficial que exhibieran el receptor 3 del factor de crecimiento de fibroblastos mutado (FGFR3) se podría reducir la frecuencia de las cistoscopias en comparación con aquellos pacientes que exhibieran el tipo salvaje (no mutado) de FGFR3m, que sí desarrollarían recurrencias $^{67}$. Moreno Sierra ha señalado que en la expresión del receptor del factor del crecimiento epidérmico (EGFR) medida por radioinmunoensayo, en el cáncer vesical de la vejiga, no se ha logrado establecer un patrón normal, y por lo tanto el EGFR no se ha encontrado útil para identificar pacientes con riesgo de progresión y muerte ${ }^{68}$. La expresión de RNAm de la epiregulina (un ligando para el factor de crecimiento epidérmico), medida mediante transcripción reversa PCR en biopsias de cánceres vesicales, se ha encontrado significativamente más alta en tumores T2-T4, y se correlacionó con una peor supervivencia ${ }^{69}$. Un nivel de factor de crecimiento de hepatocitario sérico bajo se ha asociado 
significativamente a una probabilidad de supervivencia promedio mayor en pacientes con cáncer vesical superficial ${ }^{70}$. La sobre-expresión de timidina fosforilasa (TP), que es idéntica al factor de crecimiento celular endotelial derivado de plaquetas (PD-ECGF) se ha encontrado un factor pronóstico independiente de invasión local y de metástasis en nódulos linfáticos ${ }^{71}$.

\section{OTROS MARCADORES ESTUDIADOS PARA} EL PRONÓSTICO DEL CÁNCER DE VEJIGA.

Una baja expresión tumoral de interleucina (IL)-1 alfa tumoral se ha asociado significativamente con supervivencia más corta en pacientes con tumores pobremente diferenciados. Niveles altos del mRNA del activador del plasminógeno urokinasa (uAP) se ha encontrado asociado de forma significativa con una supervivencia promedio más corta ${ }^{72}$. Los niveles plasmáticos preoperatorios de IL-6 e IL-6sR (receptor soluble de la IL6), medidos mediante ensayo con inmunoabsorbente ligado a enzima, se han encontrado asociados significativamente con el estadio local del cáncer y la existencia de metástasis, y predictores independientes de recurrencia y de la supervivencia específica por la enfermedad ${ }^{73}$.

La pérdida de expresión de la molécula de adhesión celular E-cadherina, demostrada mediante inmunotinción en especímenes de cistectomía, se ha encontrado asociada significativamente a invasividad, metástasis en nódulos linfáticos y riesgo aumentado de muerte por el cáncer de vejiga ${ }^{74,75}$, siendo un predictor independiente de pobre supervivencia ${ }^{76}$. La pérdida de la expresión de E-cadherina en carcinoma in situ, podría representar un cáncer de peor pronostico requiriendo un tratamiento temprano más agresivo ${ }^{77}$.

La pérdida focal de la inmunotinción de CD44v3 y v6 (que son dos isoformas de esta molécula de adhesión celular) se encontró asociada con un más corto intervalo libre de recurrencia en cáncer vesical en estadio Ta pero no en estadio $\mathrm{T}^{78,79}$.

Niveles séricos de sFasL (ligando Fas soluble, involucrado en la apoptosis mediada por linfocitos $\mathrm{T}$ y células natural killer contra las células tumorales) elevados, se han asociado significativamente a la recurrencia precoz del cáncer vesical ${ }^{80}$.
La metaloproteinasa (MMP)-9 y el inhibidor tisular de la MMP-2 (TIMP-2) medidos por Northern blot, se han asociado significativamente a recurrencia en cánceres vesicales superficia$\mathrm{les}^{81}$. Concentraciones urinarias de MMP-1 detectables se asociaron a tumores grado 3 con invasión en profundidad, comparados con los superficiales, y con más alta proporción de progresión y muerte por cáncer de vejiga, comparado con concentraciones indetectables ${ }^{82}$.

El nivel de expresión de clusterin de RNAm medido mediante análisis Northern blot, se ha asociado significativamente a recurrencia como factor independiente ${ }^{83}$.

La expresión de Cox-2 mediante inmunotinción de los especímenes de cistectomía se ha asociado significativamente a invasión local pero no con el pronóstico ${ }^{84}$.

La expresión del gen de la glicoproteína llamada secretina, acídica y rica en cisteína (SPARC) también llamada osteonectina y BM40, se ha asociado significativamente con peor supervivencia en los cánceres de vejiga invasivos ${ }^{85}$.

Un contaje del macrófago asociado a tumores (TAM) $\geq 67$ se ha asociado significativamente a una proporción más alta de cistectomía y metástasis a distancia e invasión vascular, comparado con un contaje más bajo $(\leq 67)$ de macrófagos asociado a tumor ${ }^{86}$.

La enzima nuclear topoisomerasa II alfa se ha asociado significativamente con alto grado de invasión de la lámina propia, y se ha señalado que en los tumores superficiales seleccionaría a pacientes en una categoría pronóstica peor ${ }^{87}$.

\section{CAPACIDAD DE LOS MARCADORES TUMO- RALES PARA PREDECIR LA RESPUESTA AL TRATAMIENTO.}

Tratamiento con Bacillus de Calmette-Guerin (BCG).

La resección endoscópica más el tratamiento con BCG de un cáncer transicional vesical T1G3, permite una proporción del $80 \%$ de supervivencia promedio libre de enfermedad a los 5 años con preservación de la vejiga ${ }^{88}$. La respuesta clínica en los 3 primeros meses al tratamiento endovesical con BCG se ha encontrado un factor predictivo de progresión a invasión en los T1G3, conduciendo a considerar una cistectomía precoz ${ }^{89}$. 
Una cistectomía precoz podría mejorar la supervivencia a largo plazo en los pacientes en los que falla el tratamiento con $\mathrm{BCG}^{90}$. En los pacientes con cáncer vesical en estadios $\mathrm{Ta} / \mathrm{T} 1$ en tratamiento con BCG, la presencia de CIS concomitante se ha asociado significativamente a progresión y peor pronóstico ${ }^{91}$.

Después de un solo curso de BCG, los niveles de IL-2 en orina inferiores a $27 \mathrm{pg} / \mathrm{micromol}$ de creatinina, se han asociado significativamente a recurrencia en comparación con niveles más altos $^{92}$.

En especímenes de cáncer vesical T1G3, una inmunorreactividad MIB-1 negativa (menor de $20 \%)$ se ha asociado significativamente con una respuesta positiva a las instilaciones de BCG. Sin embargo, MIB-1 es de uso clínico limitado por su baja especificidad, y no sirve para seleccionar candidatos para la cistectomía ${ }^{93}$.

En cánceres vesicales superficiales tratados con BCG, el índice de marcaje de Ki-67 (a un nivel de corte de $13 \%$ ) se asoció significativamente de forma independiente a progresión, pero este hallazgo no se halló correlacionado con la recurrencia $^{94}$.

La medida cuantitativa de la expresión de IL8 (>4000ng) y de IL-18 (punto de corte no establecido aún, aunque se ha señalado un promedio de $12258 \mathrm{pg}$ ) en la orina durante las primeras 6 horas y las primeras 12 horas respectivamente después de la instilación intravesical de BCG, se ha encontrado útil en predecir el riesgo de recurrencia, siendo el riesgo más bajo cuando los niveles de IL-8 e IL-18 son más en los periodos mencionados ${ }^{95}$.

En el tratamiento con Mitomicina C de un tumor con un grado mayor de 1, ni la ploidía del $\mathrm{ADN}$, ni la fracción en fase $\mathrm{S}$, se encontraron útiles para predecir las recurrencias o para aportar información pronóstica adicional al grado tumo$\mathrm{ral}^{96}$.

\section{Cistectomía radical}

El confinamiento al órgano y el estado de los nódulos linfáticos (a partir de la linfadenectomía pelviana bilateral) se han encontrado asociados significativamente a la supervivencia específica de la enfermedad en los pacientes cistectomiza$\operatorname{dos}^{97}$.
Alguna serie no ha encontrado asociación significativa entre la edad del paciente o la hidronefrosis preoperatoria y la supervivencia. Se ha descrito una supervivencia a 5 años para el tumor organoconfinado de un $44,1 \%$, situándose la supervivencia global en un $22 \%$. Se considera que la cistectomía radical es el mejor tratamiento del cáncer vesical invasivo, pero ofrece pocas posibilidades de curar al paciente. El mejor candidato para una cistectomía radical es un paciente con un tumor de grado bajo o medio y organoconfinado. En otros casos es sólo un procedimiento paliativo ${ }^{98}$.

El efecto terapéutico de la linfadenectomía no se ha documentado aún. Se ha encontrado que una linfadenectomía más extensa mejoraría significativamente el pronóstico de pacientes con cáncer vesical invasivo ${ }^{99}$.

\section{Radioterapia}

En radioterapia curativa subsiguiente a resección transuretral, se ha encontrado asociación significativa entre un índice apoptótico bajo y una positividad de p53 (considerando el punto de corte en $>20 \%$ ) y un peor control local ${ }^{100}$.

Se ha sugerido que pacientes con un índice apoptótico espontáneo alto y un índice K-67 alto antes del tratamiento, deberían ser pacientes considerados para radioquimioterapia, mientras que los pacientes con tumores con baja proliferación y bajos niveles de apoptosis es menos probable que respondan a la radioquimioterapia ${ }^{101}$.

\section{Quimioterapia}

En pacientes con cáncer de vejiga avanzado, una evaluación temprana de la respuesta ha de evitar una toxicidad innecesaria.

La inmunotinción p53-negativo/p21-positivo se ha encontrado un posible factor predictor de respuesta favorable a la quimioterapia ${ }^{102}$.

Los niveles de expresión del factor de crecimiento endotelial vascular (VEGF) y del factor de crecimiento fibroblástico básico (bFGF), medidos por hibridación fluorescente in situ, y densidad microvascular, determinada por inmunohistoquímica, se han encontrado útiles para identificar pacientes con cáncer vesical músculo invasivo, con riesgo de desarrollar metástasis después de una cistectomía radical y quimioterapia sistémica agresiva M-VAC ${ }^{103}$. 
Se han encontrado significativamente más altos los valores de linfocitos antes del tratamiento, la proporción $\mathrm{CD} 4+/ \mathrm{CD} 8+$, y el número de células natural killer, en pacientes que responden a ciertos regimenes de quimioterapia, comparados con pacientes que no responden a dicho tratamiento $^{104}$.

El 5-FU (5-fluoruracilo) es un agente anticancerígeno que inhibe la timidilato sintasa (TS). La dihidropirimidina dehidrogenasa (DPD) degrada al 5-FU. Se ha encontrado que los pacientes con baja actividad TS y alta DPD muestran el periodo más largo libre de enfermedad post-operatorio. La actividad TS elevada se ha asociado significativamente a una probabilidad más alta de progresión y recurrencia del cáncer de vejiga ${ }^{105}$.

\section{CONCLUSIONES}

Ante el hecho de que en los pacientes con cáncer de vejiga clasificados como TaG1, la proporción de supervivencia a los 5 años es similar a la población normal, a pesar de existir recurrencia del tumor hasta en un $50 \%$ de $\operatorname{casos}^{106}$ y de que por otra parte hay que considerar a los tumores papilares de vejiga, aún en estadio $\mathrm{T} 1$, sea cual sea el grado histológico, como una enfermedad con un pronóstico serio, que recomienda una estrecha vigilancia endoscópica, nos encontramos ante el gran reto de un abanico amplio de posibilidades a la hora de atentar un pronóstico e indicar un tratamiento.

Los actuales factores clinicopatológicos parecen insuficientes para predecir la recurrencia y la probabilidad de progresión. Sin embargo, la intensa investigación actual en busca de un marcador tumoral para pronosticar el cáncer de vejiga, bien basándose en una más precisa caracterización de factores nucleares, o bien a nivel molecular, por el momento no aportan datos concluyentes que puedan trasladarse a la práctica clínica.

Por una parte, hay necesidad de una estandarización de los métodos de determinación de algunos marcadores, y de un consenso en los niveles de corte para decir si la prueba es positiva o negativa, y así ser reproducible. Por otra parte, los resultados controvertidos que arrojan los estudios del mismo marcador llevados a cabo por diferentes autores, obligan a cuestionar en algunos casos su significado en cuanto al pronóstico en el cáncer vesical.
Agradecimientos. La profesora V. Lokeshwar nos ha guiado con su escrupulosa búsqueda de la verdad. El profesor R. González en su excelencia académica ha sido clave para que pudiera llevarse a cabo este trabajo. Agradecemos encarecidamente la ayuda técnica y buen hacer de Ms. D. Singh.

\section{REFERENCIAS}

1. BUSTO CATANON L, SÁNCHEZ MERINO JM, PICALLO SÁNCHEZ JA, GELABERT MAS A.: Clinical prognostic factors in superficial cancer of the urinary bladder. Arch Esp Urol 2001; 54 (2): 131-138.

2. MICHELI A, MARIOTTO A, GIORGI ROSSI A, GATTA G, MUTI P.: The prognostic role of gender in survival of adult cancer patients. Eur $J$ Cancer 1998; 34 (14): 2271-2278.

3. IORI F, DE DOMINICIS C, LIBERTI M, FRIONI D, VAHEDI M, LEONARDO C, DE NUNZIO C, LAURENTI C.: Superficial bladder tumors in patients under 40 years of age: clinical, prognostic and cytogenetic aspects. Urol Int 2001; 67 (3): 224-227.

4. HARA S, MIYAKE H, FUJISAWA M, OKADA H, ARAKAWA S, KAMIDONO S, HARA I.: Prognostic variables in patients who have undergone radical cystectomy for transitional cell carcinoma of the bladder. Jpn J Clin Oncol 2001; 31 (8): 399-402.

5. JIMÉNEZ RE, GHEILER E, OSKANIAN P, TIGUERT R, SAKR W, WOOD DP JR, PONTES JE, GRIGNON DJ.: Grading the invasive component of urothelial carcinoma of the bladder and its relationship with progression-free survival. Am J Surg Pathol 2000; 24 (7): 980-987.

6. WATTERS AD, BALLANTYNE SA, GOING JJ, GRIGOR KM, BARTLETT JM.: Aneusomy of chromosomes 7 and 17 predicts the recurrence of transitional cell carcinoma of the urinary bladder. BJU Int 2000; 85 (1): 42-47.

7. BRODERS AC.: Epithelioma of the genitourinary organs. Ann Surg 1922; 75: 574.

8. EDWARD M, MESSING, CATALONA W.: Bladder cancer. Campbell's Urology. Seventh Edition 1998; 3: 2329-2410.

9. KOSHIKAVA T, LEYH H, SCHENCK U.: Difficulties in evaluating urinary specimens after local mitomycin therapy of bladder cancer. Diagn Cytopathol 1989; 5: 117.

10. TOSONI I, WAGNER U, SAUTER G, EGLOFF M, KNONAGEL H, ALUND G, BANNWART F, MIHATSCH MJ, GASSER TC, MAURER R.: Clinical significance of interobserver differences in the staging and grading of superficial bladder cancer. BJU Int 2000; 85 (1): 48-53.

11. MATOS T, CUFER T, CERVEK J, BORNSTNAR S, KRAGELJ B, ZUMER-PREGELJ M.: Prognostic factors in invasive bladder carcinoma treated by combined modality protocol (organ-sparing approach). Int $J$ Radiat Oncol Biol Phys 2000; 46 (2): 403-409.

12. CHANG BS, KIM HL, YANG XJ, STEINBERG GD.: Correlation between biopsy and radical cystectomy in assessing grade and deph of invasion in bladder urothelial carcinoma. Urology 2001; 57 (6): 1063-1066.

13. BILLIS A, CARVALHO RB, MATTOS AC, NEGRETTI F, NOGUEIRA CR, OLIVEIRA MC, VALENCA JT JR, ADAM RL, COTTA AC, NUNES MS, DINAMARCO PV.: Tumor grade heterogeneity in urothelial bladder carcinoma: Proposal of a system using combined numbers. Scand J Urol Nephrol 2001; 35 (4): 275-279. 
14. MILLÁN-RODRÍGUEZ F, CHECHILE-TONIOLO G, SALVADOR-BAYARRI J, PALOU J, VICENTE-RODRÍGUEZ J.: Multivariate analysis of the prognostic factors of primary superficial bladder cancer. J Urol 2000; 163 (1): 73-78.

15. SHARIAT SF, WEIZER AZ, GREEN A, LAUCIRICA R, FROLOV A, WHEELER TM, LERNER SP.: Prognostic value of p53 nuclear accumulation and histopathologic features in $\mathrm{T} 1$ transitional cell carcinoma of the urinary bladder. Urology 2000; 56 (5): 735-740.

16. UCHIDA T, MINEI S, GAO JP, WANG C, SATOH T, BABA S.: Clinical significance of p53, MDM2, and bcl2 expression in transitional cell carcinoma of the bladder. Oncol Rep 2002; 9 (2): 253-259.

17. WIJKSTROM H, EDSMYR F, LUNDH B.: The value of preoperative classification according to the TNM system. Eur Urol 1984; 10: 101.

18. YOUNES M, SUSSMAN J, TRUE L.: The usefulness of the level of the muscularis mucosae in the staging of invasive transitional cell carcinoma of the urinary bladder. Cancer 1990; 66: 543.

19. PHILIP AT, AMIN MB, TAMBOLI P, LEE TJ, HILL CE, RO JY.: Intravesical adipose tissue: a quantitative study of its presence and location with implications for therapy and prognosis. Am J Surg Pathol 2000; 24 (9): 1286-1290.

20. HUGUET PÉREZ J, PALOU J, MILLAN RODRÍGUEZ F, VILLAVICENCIO MAVRICH H, RODRÍGUEZ JV.: Radical cystectomy for superficial tumors in the BCG era. Arch Esp Urol 2002; 55 (1): 50-56.

21. PANTAZOPOULOS D, IOAKIM-LIOSSI A, KARAKITSOS P, ARONI K, KAOLIRIS S, KANAVAROS P, KYRKON KA.: DNA content and proliferation activity in superficial transitional cell carcinoma of the bladder. Anticancer Res 1997; 17 (1B): 781-786.

22. CAJULIS RS, HAINES GK $3^{\text {rd }}$, FRIAS-HIDVEGI D, MCVARY K, BACUS JW.: Cytology, flow cytometry, image analysis and interphase cytogenetics by fluorescence in situ hibridization in the diagnosis of transitional cell carcinoma in bladder washes: a comparative study. Diag Cytopathol 1995; 13 (3): 214-223.

23. CIANCIULLI AM, BOVANI R, LEONARDO F, ANTENUCCI A, GANDOLFO GM, GIANNARELLI D, LEONARDO C, IORI F, LAURENTI C.: Interphase cytogenetics of bladder cancer progression: relationship between aneusomy, DNA ploidy pattern, histopathology and clinical outcome. Int $J$ Clin Lab Res 2000; 30 (1): 5-11.

24. VAN RHIJN BW, VAN DER POEL HG, BOON ME, DEBRUYNE FM, SCHALKEN JA, WITJES JA.: Presence of carcinoma in situ and high 2C-deviation index are the best predictors of invasive transitional cell carcinoma of the bladder in patients with high risk. Quanticyt 2000; 55 (3): 363-367.

25. OZER E, YORUKOGLU K, MUNGAN MU, OZKAL S, DEMIREL D, SAGOL O, KIRBA Z.: Prognostic significance of nuclear morphometry in superficial bladder cancer. Anal Quant Cytol Histol 2001; 23 (4): 251-256.

26. FUJIKAWA K, MATSUI Y, OKA H, FUKUZAWA S, SASAKI M, TAKEUCHI H.: The role of volume weighted mean nuclear volume in predicting the prognosis of patients with primary transitional cell carcinoma of the upper urinary tract: a report of 102 new cases. J Urol 2000; 164 (2): 352-355.
27. ÁLVAREZ KINDELAN J, LÓPEZ BELTRÁN A, REQUENA TAPIA MJ.: Biologia molecular en el cáncer vesical. Actas Urol Esp 2000; 24 (8): 604-625.

28. WOLF HK, STOBER C, HOHENFELLNER R, LEISSNER J.: Prognostic value of p53, p21/WAF1, Bcl-2, Bax, Bak and Ki-67 immunoreactivity in pT1G3 urothelial bladder carcinomas. Tumour Biol 2001; 22 (5): 328336.

29. MORENO SIERRA J, LUENGO ALPUENTE S, LÓPEZ GARCÍA-ASENJO J, REDONDO GONZÁLEZ E, BLANCO JIMÉNEZ E, FERNÁNDEZ PÉREZ C, SILMI MOYANO A, RESEL ESTÉVEZ L.: Usefulness of p53 oncoprotein in urinary wash cytology: experience in patients with superficial bladder carcinoma. Arch Esp Urol 1999; 52 (9): 943-954.

30. MOYANO CALVO JL, DE MIGUEL RODRÍGUEZ M, POYATO GALÁN JM, ORTIZ GAMIZ A, MOLINA CARRANZA A, ZERPA RAILEY JJ, TORO CEPEDA H, SÁNCHEZ-BARRIGA PENA D, GALERA DAVIDSON H, CASTIÑEIRAS FERNÁNDEZ J.: DNA ploidy determination with flow cytometry, Ki-index and overexpression of p53 protein in $121 \mathrm{~T} 1$ superficial bladder carcinomas. Retrospective studies. Part II: Prognostic value and usefulness in the indication for prophylactic treatment with BCG. Actas Urol Esp 2001; 25 (1): 32-45.

31. TOKTAS G, TURKERI LN, UNLUER E, ATUG F, MURAT C, OZVEREN B, CALISKAN M, AKDAS A.: Prognostic significance of p53 protein accumulation in stage pT1 transitional cell carcinoma of the bladder. Int Urol Nephrol 1999; 31 (4): 437-441.

32. GAO JP, UCHIDA T, WANG C, JIANG SX, MATSUMOTO $\mathrm{K}$, SATOH T, MINEI S, SOH S, KAMEYA T, BABA S.: Relationship between p53 gene mutation and protein expression: clinical significance in transitional cell carcinoma of the bladder. Int $J$ Oncol 2000; 16 (3): 469-475.

33. MORENO SIERRA J, LÓPEZ GARCÍA ASENJO JA, REDONDO GONZÁLEZ E, FERNÁNDEZ PÉREZ C, MAESTRO DE LAS CASAS ML, BLANCO JIMÉNEZ E, SILMI MOYANO A, RESEL ESTÉVEZ L.: Usefulness of p53 oncoprotein immunohistochemistry in the follow up of bladder carcinoma: a 5 year study. Arch Esp Urol 1999; 52 (8): 840-848.

34. LEISSNER J, HOHENFELLNER R, THUROFF JW, KOPPEN C, WOLF HK.: Prognostic significance of histopathological grading and immunoreactivity for p53 and p21/WAF1 in grade 2 pTa transitional cell carcinoma of the urinary bladder. Eur Urol 2001; 39 (4): 438-445.

35. KRUSPSKI T, MOSKALUK C, BOYD JC, THEODORESCU D.: A prospectivepilot evaluation of urinary and immunohistochemiacl markers as predictors of clinical stage of urothelial carcinoma of the bladder. BJU Int 2000; 85 (9): 1027-1032.

36. GONTERO P, CASETTA G, ZITELLA A, BALLARIO R, PACCHIONI D, MAGNANI C, MUIR GH, TIZZANI A.: Evaluation of p53 protein overexpression, Ki-67 proliferative activity and mitotic index as markers of tumor recurrence in superficial transitional cell carcinoma of the bladder. Eur Urol 2000; 38 (3): 287-296.

37. FRIEDRICH MG, RIETHDORF S, ERBERSDOBLER A, TIEMER C, SCHWAIBOLD H, SOLTER JK, HULAND E, RIETHDORF L, CONRAD S, HAMMERER PG, HULAND H.: Relevance of p53 gene alterations for tumor recurrence in patients with superficial transitional cell carcinoma of the bladder. Eur Urol 2001; 39 (2): 159-166. 
38. SCHMITZ-DRAGER BJ, GOEBELL PJ, EBERT T, FRADET Y.: p53 immunohistochemistry as a prognostic marker in bladder cancer. Playground for urology scientists? Eur Urol 2000; 38 (6): 691-699.

39. BERNARDINI S, CHABANNES E, BITTARD H. Usefulness of p53 in the clinical management of bladder tumors. Prog Urol 2001; 11 (2): 201-208.

40. ASCI R, YILDIZ L, SARIKAYA S, BUYUKALPELLI R, YILMAZ AF, KANDEMIR B.: p53 and bcl-2 overexpression as associated risk factors in patients 40 years old or less with transitional cell carcinoma of the bladder. Urol Int 2001; 67 (1): 34-40.

41. LENG J, ZHANG Y, YAO X.: Expression of bcl-2 and p16 in transitional cell carcinoma of urinary bladder. Zhonghua Wai Ke Za Zhi 2000; 38 (1): 40-43.

42. MIYAMOTO H, KUBOTA Y, NOGUCHI S, TAKASE K, MATSUZAKI J, MORIYAMA M, TAKEBAYASHI S, KITAMURA H, HOSAKA M.: c-erbB-2 gene amplification as a prognostic marker in human bladder cancer. Urology 2000; 55 (5): 679-683.

43. MORENO SIERRA J, MAESTRO DE LAS CASAS ML, REDONDO GONZÁLEZ E, FERNÁNDEZ PÉREZ C, DEL BARCO BARRIUSO V, SANZ CASLA MT, BLANCO JIMÉNEZ E, SILMI MOYANO A, RESEL ESTÉVEZ L.: p185 (Neu) oncoprotein in the prognosis on bladder carcinoma. Experience of 5 years. Arch Esp Urol 2000; 53 (3): 238-244.

44. JIMÉNEZ RE, HUSSAIN M, BIANCO FJ JR, VAISHAMPAYAN U, TABAZCKA P, SAKR WA, PONTES JE, WOOD DP JR, GRIGNON DJ.: Her-2/neu overexpression in muscle invasive urothelial carcinoma of the bladder: Prognostic significance and comparative analysis in primary and metastatic tumors. Clin Cancer Res 2001; 7 (8): 2440-2447.

45. SIMONEAU M, LA RUE H, ABOULKASSIM TO, MEYER F, MOORE L, FRADET Y.: Chromosome 9 deletions and recurrence of superficial bladder cancer: Identification of four regions of prognostic interest. Oncogene 2000; 19 (54): 6317-6323.

46. EDWARDS J, DUNCAN P, GOING JJ, GRIGOR KM, WATTERS AD, BARTLETT JM.: Loss of heterozygosity on chromosomes 11 and 17 are markers of recurrence in TCC of the bladder. $\mathrm{Br} J$ Cancer 2001; 85 (12): 1894-1899.

47. EDWARDS J, DUNCAN P, GOING JJ, WATTERS AD, GRIGOR KM, BARTLETT JM.: Identification of loci associated with putative recurrence genes in transitional cell carcinoma of the urinary bladder. $J$ Pathol 2002; 196 (4): 380-385.

48. OOSTERHUIS JW, SCHAPERS RF, JANSSEN-HEIJNEN ML, SMEETS AW, PAUWELS RP.: MIB-1 as a proliferative marker in transitional cell carcinoma of the bladder: clinical significance and comparison with other prognostic factors. Cancer 2000; 88 (11): 2598-2605.

49. WU TT, CHEN JH, LEE YH, HUANG JK.: The role of bcl-2, p53, and Ki-67 index in predicting tumor recurrence for low grade superficial transitional cell bladder carcinoma. J Urol 2000; 163 (3): 758-760.

50. SGAMBATO A, MIGALDI M, FARAGLIA B, DE ALOYSIO G, FERRARI P, ARDITO R, DE GAETANI C, CAPELLI G, CITTADINI A, TRENTINI GP.: Cyclin D1 expression in papillary superficial bladder cancer: Its association with other cell cycle-associated proteins, cell proliferation and clinical outcome. Int $J$ Cancer 2002; 97 (5): 671-678.
51. KORKOLOPOULOU P, CHRISTODOULOU P, KONSTANTINIDOU AE, THOMAS-TSAGLI E, KAPRALOS P, DAVARIS P.: Cell cycle regulators in bladder cancer: A multivariate survival study with emphasis on p27Kip1. Hum Pathol 2000; 31 (6): 751-760.

52. CHOW NH, TZAI TS, CHENG HL, LIU HS, CHAN SH, TONG YC.: The clinical value of p21WAF1/ CIP1 expression in superficial bladder cancer. Anticancer Res 2000; 20 (2B): 1173-1176.

53. MIGALDI M, SGAMBATO A, GARAGNANI L, ARDITO R, FERRARI P, DE GAETANI C, CITTADINI A, TRENTINI GP.: Loss of p21Waf1 expression is a strong predictor of reduced survival in primary superficial bladder cancers. Clin Cancer Res 2000; 6 (8): 3131-3138.

54. KORKOLOPOULOU P, KONSTANTINIDOU AE, THOMAS-TSAGLI E, CHRISTODOULOU $P$ KAPRALOS $P$, DAVARIS P.: Waf1/p21 protein expression is an independent prognostic indicator in superficial and invasive bladder cancer. Immunohistochem 2000; 8 (4): 285-292.

55. KAMAI T, TAKAGI K, ASAMI H, ITO Y, OSHIMA $\mathrm{H}$, YOSHIDA KI.: Decreasing of p27 (Kip1) and cyclin E protein levels is associated with progression from superficial into invasive bladder cancer. $\mathrm{Br} \mathrm{J}$ Cancer 2001; 84 (9): 1242-1251.

56. TAKAGI Y, TAKASHI M, KOSHIKAWA T, SAKATA T, OHSHIMA S.: Immunohistochemical demosnstration of cyclin D1 in bladder cancers as an inverse indicator of invasiveness but not an independent prognostic factor. Int J Urol 2000; 7 (10): 366-372.

57. TOMOBE M, SHIMAZUI T, UCHIDA K, AKAZA H.: AgNOR count in resting cells (resting NOR) is a new prognostic marker in invasive bladder tumor. Anal Cell Pathol 2001; 22 (4): 193-199.

58. BOZLU M, ORHAN D, BALTACI S, YAMAN O, ELHAN AH, TULUNAY O, MUFTUOGLU YZ: The prognostic value of proliferating cell nuclear antigen, Ki-67 and nucleolar organizer region in transitional cell carcinoma of the bladder. Int Urol Nephrol 2002; 33 (1): 59-66.

59. WANG S, XIA T, ZHANG Z.: Expression of VEGF and tumor angiogenesis in bladder cancer. Zhonghua Wai Ke Za Zhi 2000; 38 (1): 34-36.

60. BERNARDINI S, FAUCONNET S, CHABANNES E, HENRY PC, ADESSI G, BITTARD H.: Serum Levels of vascular endothelial growth factor as a prognostic factor in bladder cancer. J Urol 2001; 166 (4): 1275-1279.

61. CHOW NH, LIU HS, CHAN SH, CHENG HL, TZAI TS.: Expression of vascular endothelial growth factor in primary superficial bladder cancer. Anticancer Res 1999; 19 (5C): 4593-4597.

62. CREW JP, FUGGLE S, BICKNELL R, CRANSTON DW, DE BENEDETTI A, HARRIS AL.: Eukaryotic initiation factor-4E in superficial and muscle invasive bladder cancer and its correlation with vascular endothelial growth factor expression and tumour progression. $\mathrm{Br} J$ Cancer 2000; 82 (1): 161-166.

63. CHAUDHARY R, BROMLEY M, CLARKE NW, BETTS CD, BARNARD RJ, RYDER WD, KUMAR S.: Prognostic relevance of microvessel density in cancer of the urinary bladder. Anticancer Res 1999; 19 (4C): 3479-3484.

64. KORKOLOPOULOU P, KONSTANTINIDOU AE, KAVANTZAS N, PATSOURIS E, PAVLOPOULOS PM, CHRISTODOULOU P, THOMAS-TSAGLI E, DAVARIS P.: Morphometric microvascular characteristics predict prognosis in superficial and invasive bladder cancer. Virchows Arch 2001; 438 (6): 603-611. 
65. SAGOL O, YORUKOGLU K, SIS B, TUNA B, OZER E, GURAY M, MUNGAN U, KIRKALI Z.: Does angiogenesis predict recurrence in superficial transitional cell carcinoma of the bladder? Urology 2001; 57 (5): 895899.

66. CHABANNES E, BERNARDINI S, WALLARAND H, BITTARD H.: Angiogenesis in bladder: prognosis indicator and therapeutic target. Prog Urol 2001; 11 (3): 417427.

67. VAN RHIJN BW, LURKIN I, RADVANYI F, KIRKELS WJ, VAN DER KWAST TH, ZWARTHOFF EC.: The fibroblastic growth factor receptor 3(FGFR3) mutation is a strong indicator of superficial bladder cancer with low recurrence rate. Cancer Res 2001; 61 (4): 1265-1268.

68. MORENO SIERRA J, MAESTRO DE LAS CASAS ML, REDONDO GONZÁLEZ E, FERNÁNDEZ PÉREZ C, DEL BARCO BARRIUSO V, SANZ CASLA MT, BLANCO JIMÉNEZ E, SILMI MOYANO A, RESEL ESTÉVEZ L.: Epidermal growth factor receptor (EGFR) in the prognosis of bladder carcinoma. Experience of 5 years. Arch Esp Urol 2000; 53 (4): 323-331.

69. THOGERSEN VB, SORENSEN BS, POULSEN SS, ORNTOFT TF, WOLF H, NEXO E.: A subclass of HER1 ligands are prognostic markers for survival in bladder cancer patients. Cancer Res 2001; 61 (16): 6227-6233.

70. GOHJI K, NOMI M, NIITANI Y, KITAZAWA S, FUJII A, KATSUOKA Y, NAKAJIMA M.: Independent prognostic value of serum hepatocyte growth factor in bladder cancer. J Clin Oncol 2000; 18 (16): 2963-2971.

71. ARIMA J, IMAZONO Y, TAKEBAYASHI Y, NISHIYAMA K, SHIRAHAMA T, AKIBA S, FURUKAWA T. AKIYAMA S, OHI Y.: Expression of thymidine phosphorylase as an indicator of poor prognosis for patients with transitional cell carcinoma of the bladder. Cancer 2000; 88 (5): 1131-1138.

72. SEDDIGHZADEH M, STEINECK G, JANSSON O LARSSON P, WIJKSTROM H, ADOLFSSON J, PORTWOOD N, HANSSON J, LINDER S.: Low interleukinlalpha messenger RNA levels predict decreased overall survival time of patients with urinary bladder carcinoma. Br J Cancer 2001; 84 (3): 329-334.

73. ANDREWS B, SHARIAT SF, KIM JH, WHEELER TM, SLAWIN KM, LERNER SP.: Preoperative plasma levels of interleukin-6 and its soluble receptor predict disease recurrence and survival of patients with bladder cancer. J Urol 2002; 167 (3): 1475-1481.

74. BYRNE RR, SHARIAT SF, BROWN R, KATTAN MW, MORTON RA JR, WHEELER TM, LERNER SP.: ECadherin immunostaining of bladder transitional cell carcinoma, carcinoma in situ and lymph node metastases with long-term follow-up. J Urol 2001; 165 (5): 1473-1479.

75. NAKOPOULOU L, ZERVAS A, GAKIOPOULOU-GIVALOU H, CONSTANTINIDES C, DOUMANIS G, DAVARIS P, DIMOPOULOS C.: Prognostic value of E-cadherin, beta-catenin, P120ctn in patients with transitional cell bladder cancer. Anticancer Res 2000; 20 (6B): 45714578.

76. GARCÍA DEL MURO X, TORREGOSA A, MUÑOZ J, CASTELLSAGUE $X$, CONDOM E, VIGUES F, ARANCE A, FABRA A, GERMA JR.: Prognostic value of the expression of $\mathrm{E}$-cadherin and beta-catenin in bladder cancer. Eur J Cancer 2000; 36 (3): 357-362.
77. SHARIAT SF, PAHLAVAN S, BASEMAN AG, BROWN RM, GREEN AE, WHEELER TM, LERNER SP.: E-catherin expression predicts clinical outcome in carcinoma in situ of the urinary bladder. Urology 2001; 57 (1): 60-65.

78. MIYAKE H, ETO H, ARAKAWA S, KAMIDONO S, HARA I.: Over expression of CD44v8-10 in urinary exfoliated cells as an independent prognostic predictor in patients with urothelial cancer. J Urol 2002; 167 (3): 1282-1287.

79. TOMA V, HAURI D, SCHMID U, ACKERMANN D, MAURER R, ALUND G, KNONAGEL H, RIST M, GASSER TC, SAUTER G, ROTH J.: Focal loss of CD44 variant protein expression is related to recurrence in superficial bladder carcinoma. Am J Pathol 1999; 155 (5): 1427-1432.

80. MIZUTANI Y, HONGO F, SATO N, OGAWA O, YOSHIDA O, MIKI T.: Significance of serum soluble Fas ligand in patients with bladder carcinoma. Cancer 2001; 92 (2): 287-293.

81. HARA I, MIYAKE H, HARA S, ARAKAWA S, KAMIDONO S.: Significance of matrix metalloproteinases and tissue inhibitors of metalloproteinase expression in the recurrence of superficial transitional cell carcinoma of the bladder. $J$ Urol 2001; 165 (5): 1769 1772.

82. NAKOPOULOU L, GAKIOPOULOU H, ZERVAS A, GIANNOPOULOU I, CONSTANTINIDES C, LAZARIS AC, LIAPIS H, KYRIAKOU G, DIMOPOULOS C.: MMP3mRNA and MMP-3 and MMP-1 proteins in bladder cancer: A comparison with clinicopathologic features and survival. Appl Immunohistochem Mol Morphol 2001; 9 (2): 130-137.

83. MIYAKE H, GLEAVE M, KAMIDONO S, HARA I.: Overexpression of clusterin in transitional cell carcinoma of the bladder is related to disease progression and recurrence. Urology 2002; 59 (1): 150-154.

84. SHIRAHAMA T, ARIMA J, AKIBA S, SAKAKURA C.: Relation between cyclooxigenase- 2 expression and tumor invasiveness and patient survival in transitional cell carcinoma of the urinary bladder. Cancer 2001; 92 (1): 188-193.

85. YAMANAKA M, KANDA K, LI NC, FUKUMORI T, OKA N, KANAYAMA HO, KAGAWA S.: Analysis of the gene expression of SPARC and its prognostic value for bladder cancer. J Urol 2001; 166 (6): 2495-2499.

86. HANADA T, NAKAGAWA M, EMOTO A, NOMURA T, NASU N, NOMURA Y.: Prognostic value of tumor-associated macrophage count in human bladder. Int $J$ Urol 2000; 7 (7): 263-269.

87. NAKOPOULOU L, ZERVAS A, LAZARIS AC, CONSTANTINIDES C, STRAVODIMOS C, DAVARIS P, DIMOPOULOS C.: Predictive value of topoisomerase II alpha immunostaining in urothelial bladder carcinoma. $J$ Clin Pathol 2001; 54 (4): 309-313.

88. PATARD J, MOUDOUNI S, SAINT F, RIOUXLECLERCQ N, MANUNTA A, GUY L, BALLANGER P, LANSON Y, HAJRI M, IRANI J, GUILLE F, BEURTON D, LOBEL B, BERNAR LOBEL and The members of the Groupe Necker.: Tumor progression and survival in patients with T1G3 bladder tumors: Multicentric retrospective study comparing 94 patients treated during 17 years. Urology 2001; 58 (4): 551-556. 
89. SOLSONA E, IBORRA I, DUMONT R, RUBIO-BRIONES J, CASANOVA J, ALMENAR S.: The 3-month clinical response to intravesical therapy as a predictive factor for progression in patients with high risk superficial bladder cancer. J Urol 2000; 164 (3Pt1): 690-691.

90. HERR HW, SOGANI PC.: Does early cystectomy improve the survival of patients with high risk superficial bladder tumors? J Urol 2001; 166 (4): 1296-1299.

91. TAKASHI M, WAKAI K, HATTORI T, FURUHASHI K, ONO Y, OHSHIMA S, OHNO Y.: Multivariate evaluation of factors affecting recurrence, progression, and survival in patients with superficial bladder cancer treated with intravesical bacillus Calmette-Guerin (ToKyo 172 strain) therapy: Significance of concomitant carcinoma in situ. Int Urol Nephrol 2002; 33 (1): 41-47.

92. SAINT F, PATARD JJ, MAILLE P, SOYEUX P, HOZNEK A, SALOMON L, ABBOU CC, CHOPIN DK.: Prognostic value of a $\mathrm{T}$ helper 1 urinary cytokine response after intravesical bacillus Calmette-Guerin treatment for superficial bladder cancer. J Urol 2002; 167 (1): 364 367.

93. LEBRET T, BECETTE V, HERVE JM, MOLINIE V, BARRE P, LUGAGNE PM, GENTILLE A, BAGLIN AC, BOTTO H.: Prognostic value of MIB-1 antibody labeling index to predict response to Bacillus Calmette-Guerin therapy in a high risk selected population of patients with stage T1 grade G3 bladder cancer.

94. BLANCHET P, DROUPY S, ESCHWEGE P, VIELLEFOND A, PARADIS V, PICHON MF, JARDIN A, BENOIT G.: Prospective evaluation of Ki-67 labeling in predicting the recurrence and progression of superficial bladder transitional cell carcinoma. Eur Urol 2001; 40 (2): 169-175.

95. THALMANN GN, SERMIER A, RENTSCH C, MOHRLE K, CECCHINI MG, STUDER UE.: Urinary Interleukin 8 and 18 predict the response of superficial bladder cancer to intravesical therapy with Bacillus CalmetteGuerin. J Urol 2000; 164 (6): 2129-2133.

96. DE VERE WHITE RW, DEITCH AD, DANESHMAND S, BLUMENSTEIN B, LOWE BA, SAGALOWSKY AI, SMITH JA JR, SCHELLHAMMER PF, STANISIC TH, GROSSMAN HB, MESSING E, CRISSMAN JD, CRAWFORD ED.: The prognostic significance of S-phase analysis in stage Ta/T1 bladder cancer. A Southwest Oncology Group Study. Eur Urol 2000; 37 (5): 595600.

97. GSCHWEND JE, DAHM P, FAIR WR.: Disease specific survival as endpoint of outcome for bladder cancer patients following radical cystectomy. Eur Urol 2002; 41 (4): 440-448.

98. SLOJEWSKI M: Results of radical cystectomy for management of invasive bladder cancer with special reference to prognostic factors and quality of life depending on the type of urinary diversion. Ann Acad Med Stetin 2000; 46: 217-229.

99. LEISSNER J, ALLHOFF EP, HOHENFELLNER R, WOLF HK.: Significance of pelvic lymphadenectomy for the prognosis after radical cystectomy. Zentralbl Chir 2002; 127 (4): 315-321.

100. ONG F, MOONEN LM, GALLEE MP, TEN BOSCH C, ZERP SF, HART AA, BARTELINK H, VERHEIJ M.: Prognostic factors in transitional cell cancer of the bladder: an emerging role of Bcl-2 and p53. Radiother Oncol 2001; 61 (2): 169-175.

101. RODEL C, GRABENBAUER GG, RODEL F, BIRKENHAKE S, KUHN R, MARTUS P, ZORCHER T, FURSICH D, PAPADOPOULOS T, DUNST J, SCHROTT KM, SAUER R: Apoptosis, p53, bcl-2 and Ki-67 in invasive bladder carcinoma: Possible predictors for response to radiochemotherapy and successful bladder preservation. Int $J$ Radiat Oncol Biol Phys 2000; 46 (5): 1213-1221.

102. KOGA F, KITAHARA S, ARAI K, HONDA M, SUMI S, YOSHIDA K.: Negative p53/positive p21 immunostaining is a predictor of favorable response to chemotherapy in patients with locally advanced bladder cancer. Jpn J Cancer Res 2000; 91 (4): 416-423.

103. INOUE K, SLATON JW, KARASHIMA T, YOSHIKAWA C, SHUIN T, SWEENEY P, MILLIKAN R, DINNEY CP.: The prognostic value of angiogenesis factor expression for predicting recurrence and metastasis of bladder cancer after neoadjuvant chemotherapy and radical cystectomy. Clin Cancer Res 2000; 6 (12): 48664873.

104. SOYGUR T, BEDUK Y, BALTACI S, YAMAN O, TOKGOZ G.: The prognostic value of peripheral blood lymphocyte subsets in patients with bladder carcinoma treated using neoadjuvant M-VEC chemotherapy. BJU Int 1999; 84 (9): 1069-1072.

105. MIZUTANI Y, WADA H, OGAWA O, YOSHIDA O, FUKUSHIMA M, NONOMURA N, MIKI T.: Prognostic significance of thymidylate synthase activity in bladder carcinoma. Cancer 2001; 92 (3): 510-518.

106. MARBERGER M, STOECKLE M, PASANDORO V, STERNBERG CN.: Management and outcome in invasive and locally advanced bladder cancer. Eur Urol 2001; 40/5 (Curric Urol 1-9).

Dra. M.F. Lorenzo Gómez

Department of Urology

P.O. Box 016960 (M814)

Miami, Florida 33101

(Trabajo recibido el 4 septiembre de 2002) 